\title{
Avaliação externa das escolas em Portugal: Ensaio sobre racionalidade e significado ${ }^{1}$
}

\author{
External Evaluation of School in Portugal: essay \\ on rationality and meaning
}

\section{Joana Sousa²}

\section{Resumo}

A avaliação é um processo que agrega diferentes dimensões de um objeto. Em educação, e pelas características que fundamentam as Ciências Sociais, a avaliação educacional desenvolve-se com base na análise crítica do processo e dos resultados qualitativos e quantitativos, justificando o processo deliberativo do estudo do objeto com fundamentações epistemológicas e científicas, que consideram as características do contexto como uma das principais variáveis a observar. 0 processo de avaliação é complexo e, por isso, exigente do ponto de vista da criação de critérios e, consequentemente, da assunção de resultados que forneçam dados sobre o estado das políticas, dos processos e das práticas em educação. Esta dificuldade não deve ser justificativa do laissez-faire, mas promotora de um debate em torno da avaliação que permita a construção de padrões de avaliação educacional próximos das práticas educativas, dos processos e das políticas em

\footnotetext{
${ }^{1}$ Este trabalho é financiado por fundos nacionais através da FCT - Fundação para a Ciência e a Tecnologia, I.P., no âmbito do projeto PTDC/CED-EDG/30410/2017 e dos projetos UID/CED/01661/2019, Instituto de Educação, Universidade do Minho, através de fundos nacionais da FCT/MCTES-PT, CIEd - Centro de Investigação em Educação, Instituto de Educação, Universidade do Minho.

2 Doutora em Ciência da Educação, Investigadora do Centro de Investigação em Educação (CIEd), Universidade do Minho (UMinho), Braga, Portugal; ORCID: 0000-0002-6911-7413; Email: jsousa@ie.uminho.pt
} 
educação e respeitadoras dos seus contextos. $\mathrm{O}$ objetivo deste ensaio é refletir sobre os processos de avaliação educacional no contexto Português, focado na análise da experiência da Avaliação Externa das Escolas (AEE).

Palavras chave: Avaliação Externa das Escolas; Avaliação Educacional; Ciência; Ética.

\section{Abstract}

Evaluation is a process that aggregates different dimensions of an object. In education, and due to the characteristics that underlie the Social Sciences, educational evaluation is developed based on a critical analysis of the process and qualitative and quantitative results, justifying the deliberative process of studying the object with epistemological and scientific foundations, which consider the context characteristics as one of the main variables to observe. The evaluation process is complex and, therefore, demanding for the creation of criteria and, consequently, of the assumption of results that provide data on the state of the policies, processes, and practices in education. This difficulty should not be a justification for laissez-faire but be a promoter of the debate around evaluation that allows the construction of educational evaluation standards close to educational practices, processes, and policies in education and respectful of their contexts. The purpose of this essay is to reflect on the educational evaluation processes in the Portuguese context, focused on the analysis of the experience of the External Evaluation of Schools (EES).

Keywords: External Evaluation of School; Educational Evaluation; Science; Ethics.

\section{Introdução}

No contexto português, a aproximação entre a ciência e a educação é fundamental para a observação, análise e compreensão dos processos de avaliação educacional, contribuindo com conhecimento científico para o desenvolvimento de 
uma escola ${ }^{3}$ autónoma, democrática e inovadora, tal como é preconizado no Art. ${ }^{0}$ $50 .^{\circ}$ da Lei n. ${ }^{\circ}$ 46/86 - Lei de Bases do Sistema Educativo.

Apesar de em teoria o apelo à avaliação educacional ser uma forma de melhorar o capital educacional - e tal como Hargreaves e Fullan (2012) especificam: o 'capital profissional'-, estimulando a inovação em educação (Pascual, 2019; Vincent-Lancrin, Urgel, Kar e Jacotin, 2019), as interpretações e os resultados de vários estudos realizados em Portugal, nomeadamente sobre o sistema de Avaliação Externa das Escolas (AEE) (Barreira, Bidarra e Vaz-Rebelo, 2016; Pacheco, 2014; Pacheco, Morgado e Sousa, 2020), têm considerável relevância para a maioria dos sistemas educativos, pois alertam para a existência de várias lacunas que necessitam de uma solução para que a avaliação educacional possa cumprir as suas hipóteses teóricas, evitando vazios de significado. Este ensaio tem como objetivo apresentar uma reflexão sobre os processos de avaliação educacional no contexto Português, centrando-se na análise da experiência da AEE.

Num campo que atualmente se está a desdobrar em múltiplas conceções epistemológicas, a ciência como ética (Calado, 2016) deve aliar-se às intenções políticas, aos processos e às práticas, tornando-se um conceito central da avaliação educacional, restaurando a agência dos atores educativos (Bergh e Wahlström, 2018; Coburn, 2016; Eteläpelto, Vähäsantanen, Hökkä e Paloniemi, 2013; Imants e Van der Wal, 2019; Leijen, Pedaste e Lepp, 2019; Priestley, Biesta e Robinson, 2015) e, consequentemente, o papel da educação na sociedade.

\section{A Avaliação Educacional em Portugal}

Em 1986, com a publicação da Lei de Bases do Sistema Educativo, são definidas orientações para o desenvolvimento e avaliação do sistema educativo português, estando identificados sete eixos fundamentais: Desenvolvimento curricular (Art. 47. $^{\circ}$ ); Ocupação dos tempos livres e desporto escolar (Art.0 48. ${ }^{\circ}$ ); Avaliação do sistema educativo (Art.0 49.); Investigação em educação (Art.o 50.º);

\footnotetext{
${ }^{3}$ Neste texto as referências às escolas remetem-se a todas escolas portuguesas, quer sejam públicas ou privadas.
} 
Estatísticas da educação (Art. ${ }^{0}$ 51. ${ }^{\circ}$ ); Estruturas de apoio (Art. ${ }^{0}$ 52. ${ }^{\circ}$ ); e Inspeção escolar (Art. ${ }^{53 .{ }^{\circ}}$ ). Segundo o Art. 0 49.0 "o sistema educativo deve ser objeto de avaliação continuada, que deve ter em conta os aspetos educativos e pedagógicos, psicológicos e sociológicos, organizacionais, económicos e financeiros e ainda os de natureza político-administrativa e cultural.".

Esta perspetiva de avaliação educacional em Portugal deu lugar a várias iniciativas de avaliação das escolas inspiradas em modelos internacionais (Sousa, 2019) que incidiram, não só no conhecimento das práticas da escola, mas também no sucesso das aprendizagens, tornando os dados (Art. ${ }^{\circ} 51 .^{\circ}$ ) e o papel da Inspeção escolar, definido como "avaliar e fiscalizar a realização de educação escolar, tendo em vista a prossecução dos fins e objetivos estabelecidos na presente lei e demais legislação complementar" (Art. ${ }^{\circ}$ 53. ${ }^{\circ}$ ), essenciais para a consecução da avaliação educacional. A ausência de informação sobre os produtos, práticas e programas em educação, bem como a inexistência de sistemas que produzam essa informação, são dois dos vetores que contribuem para a inadequação da avaliação educacional (Worthen e Sanders, 1987), ficando claro que a plenitude da apropriação do conceito de avaliação nas políticas educacionais e práticas escolares, que surgiram posteriormente em Portugal, se deve ao facto de na intencionalidade política terem sido, desde logo, criadas as oportunidades para reduzir a ineficácia da avaliação do sistema educativo e a sua legitimidade junto da sociedade, acompanhando a tendência internacional: "A avaliação ganhou ampla aceitação na educação e áreas afins" (Worthen e Sanders, 1987, p.4).

\subsection{A Avaliação Externa das Escolas em Portugal}

A abordagem da avaliação educacional adotada em Portugal levou à concretização da Lei n. 0 31/2002, de 20 de dezembro, alterada pelo Art. ${ }^{\circ} 182 .^{\circ}$ da Lei n.0 66-B/2012, de 31 de dezembro, que define o Sistema de Avaliação da Educação e do Ensino não Superior, determinando

que o controlo de qualidade se deve aplicar a todo o sistema educativo com vista à promoção da melhoria, da eficiência e da eficácia, da 
responsabilização e da prestação de contas, da participação e da exigência, e de uma informação qualificada de apoio à tomada de decisão. Nos termos da lei, a avaliação estrutura-se com base na autoavaliação, a realizar em cada escola ou agrupamento de escolas, e na avaliação externa (InspeçãoGeral da Educação e Ciência, 2008, para. 1).

Criando a possibilidade de dotar os atores educativos de informação relevante para melhorar as suas práticas educativas, a AEE veio enfatizar as dimensões interna e externa, como elementos integrantes da avaliação educacional. A Inspeção-Geral da Educação e Ciência (IGEC) - entidade responsável pela implementação do modelo de AEE - criou um sistema de avaliação que corresponde às intenções previstas na Lei de Bases do Sistema Educativo, tendo em conta que a avaliação do sistema educativo "incide, em especial, sobre o desenvolvimento, regulamentação e aplicação da presente lei" (Art.0 49.0, Lei n.0 46/86, de 14 de outubro).

\section{A Avaliação Externa das Escolas em Portugal: da racionalidade ao significado}

Devido ao papel de avaliador ser uma função pela qual todas as pessoas -em algum momento da sua vida- já passaram, sendo um comportamento básico do ser humano (Worthen e Sanders, 1987), quando estamos a falar de avaliação educacional é fundamental que a ciência como ética (Calado, 2016) seja parte integrante de todo o processo de avaliação.

Sendo a AEE um sistema que avalia a qualidade das escolas, concretizando a publicação de um relatório de avaliação "composto pelas evidências e juízos avaliativos sobre cada um dos campos de análise, que suportam a classificação atribuída ao domínio [insuficiente, suficiente, bom, muito bom, excelente], bem como os pontos fortes e áreas de melhoria, tendo como principal objetivo informar e apoiar as comunidades educativas e a administração" (Inspeção-Geral da Educação e Ciência, 2019), este é um processo com vários efeitos para as escolas, quer ao nível organizacional, curricular e pedagógico - sendo que o impacto nesta última dimensão é reconhecido como reduzido ou inexistente no $2 .{ }^{\circ}$ ciclo de AEE 
(Pacheco, 2016) - entende-se que as estratégias definidas para o $3 .^{\circ}$ ciclo da AEE - como é o caso da composição da equipa de avaliação que passa a ser constituída por dois inspetores da IGEC e por dois peritos externos (Inspeção-Geral da Educação e Ciência, 2019) - permitem a melhoria deste sistema. Não obstante, para que a AEE continue a evoluir para um sistema de diferentes dimensões (interna pela autoavaliação - e externa) com o objetivo de conhecer o valor do seu objeto (Stake, 2006), tornando-se cada vez menos subjetivo - e porque a elaboração do relatório é uma das fases da avaliação mais permeáveis às questões da ética e com tendência para ter consequências mais amplas (Morris, 2003) -, deve servir-se da ciência como ética (Calado, 2016), permitindo "dar respostas a questões avaliativas significativas" (Worthen e Sanders, 1987, p. 5), por via de conhecimento validado empiricamente, valorizando o seu papel no campo da educação.

Trata-se de encontrar os fundamentos científicos para alinhar a avaliação educacional com os valores fundamentais da educação, onde a ética tem um papel preponderante, pela necessidade de desenvolver ferramentas conceptuais e metodológicas para analisar a avaliação educacional em diversos contextos sociais, políticos e culturais (Bazzul, 2017). Aliás, Stufllebeam (2003, p. 282) defende que na avaliação educacional a contextualização "exige que as avaliações sejam conduzidas legalmente, eticamente, e levando em consideração o bem-estar das pessoas envolvidas no processo, bem como as afetadas pelos resultados". Ainda que longa, obscura e lenta, "na ciência há esta coisa muito bela: é mais fácil apontar o erro do que provar a verdade" (Calado, 2016, p. 4) e, por isso, é através da sistematização da análise da observação e da experiência que é possível reconhecer o erro e avançar. A ciência ensina-nos a olhar o mundo na sua complexidade e, tal como Russell (2009, p. 533) defende: "não te sintas absolutamente certo de nada", pois a construção de conhecimento faz-se quando se é capaz de colocar as nossas convicções em causa e refutá-las, se necessário. Dado que é mais fácil identificar o erro do que provar a verdade, não devemos considerar a crítica proveniente da avaliação das escolas como uma ameaça, nem simplesmente querer que o processo de avaliação confirme o que já é do conhecimento prévio ou que sirva de justificação 
para resolver problemas internos (Morris, 2003), permanecendo-se pelo efeito de legitimação (Pacheco, 2016). Deve-se, então, considerar a avaliação como uma pista válida em busca da conceptualização da realidade.

$\mathrm{Na}$ avaliação das escolas, o papel do avaliador foca-se em interpretar a 'conduta da conduta', pelo que está intrinsecamente relacionada com o poder exercido sobre os sujeitos 'livres', numa tentativa de produção de significado de um contexto complexo, para dar resposta às necessidades conceptuais do ser humano (Foucault, 1982). Só que esta "conceptualização implica pensamento crítico - uma verificação constante" (Foucault, 1982, p. 778) fundada num determinado enquadramento teórico e numa dada realidade (Foucault, 1982). O pensamento científico implica raciocínio lógico e lateral, procurando semelhanças e diferenças, consensos e divergências mesmo quando consideramos que já sabemos tudo:

Todos estamos cientes de tais factos banais. Mas o facto de serem banais não significa que não existem. O que temos a fazer com os factos banais é descobrir - ou tentar descobrir - qual o problema específico e talvez original que esteja relacionado com eles (Foucault, 1982, p. 779).

A AEE necessita de ser legitimada pelos atores que desenvolvem as práticas escolares. Numa investigação realizada no contexto americano (Warren e Ward, 2017) torna-se claro, como em muitos outros estudos de investigação nacionais (Barreira, Bidarra e Vaz-Rebelo, 2016; Correia, 2012; Correia, 2016; Figueiredo, 2017; Gomes, 2014; Oliveira, 2017; Sampaio, 2018; Silvestre, 2013; Sousa, 2019) e internacionais (Altrichter e Kemethofer, 2015; Bonner, Rivera e Chen, 2018; Ehren, Altrichter, McNamara e O'Hara, 2013; Ehren, Gustafsson, Altrichter, Skedsmo, Kemethofer e Huber, 2015; Ehren e Visscher, 2006; Gustafsson, Ehren, Conyngham, McNamara, Altrichter e O'Hara, 2015; Hult e Edström, 2016; Jones et al., 2017; Kemethofer, Gustafsson e Altrichter, 2017; Penninckx, 2017; Penninckx e Vanhoof, 2015; Quintelier, Vanhoof e De Maeyer, 2018) que os atores educativos sentem pressão e responsabilidade nos processos de avaliação, levando-os, frequentemente, a experienciar a desconexão entre as intenções políticas e o resultado desses processos de avaliação. Para contrariar essa tendência é urgente 
que a AEE tenha significado para aqueles que se encontram ao nível das práticas escolares, como é o caso dos docentes que "desejam uma avaliação significativa (em oposição à avaliação onesize-fits-all) e instrumentos de avaliação baseados em investigação" (Warren e Ward, 2017, p. 15). A confiança entre políticas, processos e práticas de avaliação gera-se com base na ciência como ética (Calado, 2016) e no foco formativo da avaliação educacional em detrimento do foco nos resultados, dado que, por exemplo, os docentes "não resistem às reformas per se, mas a construções particulares da reforma que excluem a participação dos professores e negam a singularidade da experiência individual por uma questão de uniformidade" (Warren e Ward, 2017, p. 16). O contexto local na avaliação educacional é um dos pontos-chave dos processos de avaliação. Evitar a conformidade na avaliação educacional é dar asas à transformação e ao desenvolvimento. Apesar do conceito de inovação não ser consensual (Pascual, 2019), podemos sublinhar que a inovação e a mudança em contexto educacional deverão ter como alicerces a ciência como ética (Calado, 2016), para que a AEE não seja estéril. Na esteira de Foucault (1982), o que nos parece ser fundamental é "analisar racionalidades específicas, em vez de sempre invocar o progresso da racionalização em geral". A ciência como ética (Calado, 2016), como primazia da avaliação das escolas, recorre ao cruzamento de conhecimentos (Vo e Christie, 2018), despoletando descobertas, como é o caso da física, da química e da biologia que demonstraram que as fronteiras entre saberes são ténues, podendo residir nestes (re)encontros a inovação: "é nas fronteiras entre duas porções homogéneas de matérias gasosa, líquida ou sólida que a matéria é mais energética e reativa" (Calado, 2016, p. 11). Apesar deste ser um exemplo das ciências 'duras', a 'liquidez' das ciências sociais - como o é a educação -, deve ser um motivo para que a ciência como ética (Calado, 2016) seja vista como um contributo para a riqueza da sua complexidade, pois "o objetivo final da ciência é a unificação e a universalização: mostrar as semelhanças entre coisas diferentes" (Calado, 2016, p. 9).

Consequentemente, a ciência como ética (Calado, 2016) nos processos de avaliação educacional permite a obtenção de evidências para a construção de 
significado, devendo ser uma preocupação quer dos académicos, quer da sociedade em geral, no sentido de servir para orientar organismos, consultores e formuladores de políticas e facilitar a comunicação entre uns e outros (Building Evidence in Education, s/d), gerando os debates e consensos necessários para que o conhecimento não seja enviesado e beneficie a todos (Gorard, 2020). Como recorda Foucault (1997, p. 158):

Vivemos num mundo relacional consideravelmente empobrecido pelas instituições. A sociedade e as instituições que a estruturam limitaram a possibilidade de relacionamentos, porque um mundo relacional rico seria muito complexo de gerir. Deveríamos lutar contra o empobrecimento do tecido relacional. Devemos garantir o reconhecimento por relações de coexistência provisória, adoção...

$\mathrm{Na}$ avaliação educacional o desenvolvimento de padrões, princípios e/ou códigos tem funções específicas relevantes, sendo fundamental para o que Merton (1973, p. 267) descreve como "a estrutura normativa da ciência". A consciência científica da avaliação educacional permite a Stufflebeam (2003) argumentar que: i) protege o indivíduo e a sociedade de práticas prejudiciais; ii) responsabiliza os prestadores de serviços; iii) analisa os serviços prestados; iv) julga reivindicações de negligência; v) garante a ação com base nas melhores práticas disponíveis; vi) identifica necessidades de melhoria tecnológicas; vii) fornece um enquadramento teórico-concetual para orientar a investigação e a ação; viii) define princípios gerais para abordar uma variedade de questões práticas; ix) cria uma linguagem comum para facilitar a comunicação e colaboração; x) facilita conteúdo essencial para a formação dos atores educativos; e, por último, xi) permite ganhar e manter a confiança do público na prática. O carácter útil, exequível, contextualizado e preciso (Stufflebeam, 2003) da AEE está dependente do entendimento educacional considerado previamente às políticas educativas serem implementadas. Se o racional da $A E E$ estiver intrinsecamente focado no facto de que "a avaliação não deve apenas relatar feedback sobre pontos fortes e fracos, mas também deve ajudar os usuários a estudar e aplicar as descobertas" (Stufflebeam, 2003, p. 281), a ciência como ética (Calado, 2016) pode permitir a geração de processos de 
melhoria enraizados em cada contexto.

Num artigo publicado na Revista Nature (Shah, 2020), afirma-se que os desafios globais que se irão sentir nesta década precisam das ciências sociais. Os benefícios da avaliação educacional para o desenvolvimento da sociedade do século XXI não devem ser negligenciados. Hoje, tal como no passado (Worthen e Sanders, 1987), nos países desenvolvidos mantém-se a perspetiva de que a educação é um meio de resolução dos problemas sociais, não se devendo focar demasiado nas "inadequações das escolas a ponto de produzir pessimismo sobre a possibilidade de uma melhoria educacional genuína" (Worthen e Sanders, 1987, p. 3). Importa, através da ciência como ética (Calado, 2016), construir um conhecimento mais profundo e sistemático do estado da educação, quer na esfera das políticas, quer no âmbito das práticas, estimulando processos de desenvolvimento mais significativos e capazes de trazer maior evolução para a educação e, consequentemente, para a sociedade. A aproximação da ciência à educação, quer na análise das práticas e políticas educativas, quer na tomada de decisão, é essencial na conjuntura atual e "mais poderia ser feito para conectar a comunidade política aos conhecimentos externos de ciências sociais e humanas . . . [sendo que] governos sábios encontrarão maneiras de incorporar esse insight" (Shah, 2020, p. 295). Potenciar processos de avaliação educacional sistemática nas escolas - quer pela avaliação externa, quer pela autoavaliação - concedendo-Ihes os recursos necessários (humanos e materiais), é um sistema que consome um conjunto de bens, mas que é sobretudo resultado de uma visão de futuro a longo prazo e que, por isso, deve merecer toda a atenção e esforço do sistema político. Torna-se, até, difícil encontrar melhor investimento dos recursos do presente que não na educação, trave-mestra dos alicerces da humanidade.

Para continuar a aumentar a validade e confiabilidade de processos como a AEE em Portugal, é necessário aprofundar o debate em torno das descobertas válidas e significativas focadas na avaliação e análise do seu impacto, atravessando fronteiras e propondo estratégias (Vo e Christie, 2018) que permitam concretizar o que se encontra preconizado na Lei de Bases do Sistema Educativo: a investigação 
em educação (Art. ${ }^{0}$ 50. ${ }^{\circ}$ ) como eixo fundamental para orientar e avaliar o sistema educativo português.

\section{Considerações Finais}

A presente reflexão visa destacar a necessidade de identificar as imperfeições, ainda manifestamente intactas, da avaliação das escolas e o seu impacto no desenvolvimento da melhoria da qualidade da educação. A natureza da investigação aplicada orienta a consecução deste desígnio, uma vez que as problemáticas relacionadas com a AEE podem considerar-se através da constante tensão e construção partilhada pelos diversos sujeitos (individuais e coletivos) na arena.

Os contributos para esta reflexão não serão, certamente, tão diretos quanto se possa desejar, contudo a sua ausência seria indutora, por si só, da necessidade de aprofundar conhecimentos e lançar desafios que tenderão a amadurecer a avaliação das escolas. Por um lado, a ciência como ética (Calado, 2016) enriquecerá essa representação, evitando interpretações pouco fundamentadas. Por outro lado, o debate em torno da ciência como ética (Calado, 2016) na avaliação de escolas, nomeadamente no sistema de AEE em Portugal, permitirá a análise dos desafios que se afiguram mais relevantes, e não meramente refletir sobre um subconjunto da miríade de problemas éticos relacionados com a avaliação educacional. É neste sentido, que a estruturação da AEE no campo da avaliação educacional está relacionada com a perspetiva científica do seu valor empírico.

\section{Referências bibliográficas}

Altrichter, H. e Kemethofer, D. (2015). Does accountability pressure through school inspections promote school improvement? School Effectiveness and School Improvement, 26(1), 32-56. doi: 10.1080/09243453.2014.927369

Barreira, C., Bidarra, M. G. e Vaz-Rebelo, M. P. (Org.) (2016). Estudos sobre avaliação externa de escolas. Porto: Porto Editora. 
Bazzul, J. (2017) The 'subject of ethics' and educational research OR Ethics or politics? Yes please! Educational Philosophy and Theory, 49(10), 995-1005. doi: $10.1080 / 00131857.2016 .1270184$

Bergh, A. e Wahlström, N. (2018). Conflicting goals of educational action: a study of teacher agency from a transactional realism perspective. The Curriculum Journal, 29(1), 134-149. doi: 10.1080/09585176.2017.1400449

Bonner, S. M., Rivera, C. T. e Chen, P. P. (2018). Standards and assessment: coherence from the teacher's perspective. Education Assessment, Evaluation and Accountability, 30(1), 71-92. doi: 10.1007/s11092-017-9272-2

Building Evidence in Education. (s/d). Generating Evidence in the Education Sector. Disponível em https://bit.ly/3hWCS2L

Calado, J. (2016). A ciência como ética. XXI, Ter opinião, 6, 3-16.

Coburn, C. E. (2016). What's policy go to do with it? How the structure - agency debate can illuminate policy implementation. American Journal of Education, 132(3), 465-475. doi: 10.1086/685847

Correia, A. P. (2016). A avaliação das escolas: efeitos da avaliação externa nas dinâmicas de autoavaliação da escola (Tese de Doutoramento, Universidade de Évora, Portugal). Disponível em http://hdl.handle.net/10174/18552

Correia, S. (2012). Dispositivo de Autoavaliação de Escola: entre a lógica do controlo e a lógica da regulação (Tese de Doutoramento, Universidade do Minho, Braga, Portugal). Disponível em http://hdl.handle.net/1822/19723

Ehren, M. C. M., Gustafsson, J. E., Altrichter, H., Skedsmo, G., Kemethofer, D. e Huber, S. G. (2015). Comparing effects and side effects of different school inspection systems across Europe. Comparative Education, 51(3), 275-400. doi: 10.1080/03050068.2015.1045769

Ehren, M. C. e Visscher, A. J. (2006). Towards a theory on the impact of school inspection. British Journal of Educational Studies, 54(1), 51-72. doi: 10.1111/j.1467-8527.2006.00333.x

Ehren, M. C., Altrichter, H., McNamara, G. e O'Hara, J. (2013). Impact of school inspections on improvement of schools-describing assumptions on causal 
mechanisms in six European countries. Educational Assessment Evaluation and Accountability, 25(1), 3-43. doi: 10.1007/s11092-012-9156-4

Eteläpelto, A., Vähäsantanen, K., Hökkä, P. e Paloniemi, S. (2013). What is agency? Conceptualizing professional agency at work. Educational Research Review, 10, 45-65. doi: 10.1016/j.edurev.2013.05.001

Figueiredo, C. (2017). Avaliação de escolas: políticas, conceitos, processos e impactos. (Tese de Doutoramento, Universidade do Porto). Disponível em https://bit.ly/2V9AjR9

Foucault, M. (1982). The subject and power. Critical Inquiry, 8, 777-795.

Foucault, M. (1997). On the genealogy of ethics: An overview of a work in progress. In P. Rabinow (Eds.), Ethics: Subjectivity and truth (Essential Works of Foucault, 1954-1984, Vol. 1) (pp. 253-280). New York, NY: The New Press.

Gomes, S. (2014). Autoavaliação em escolas do Alentejo. Constrangimentos e oportunidades (Tese de Doutoramento, Universidade de Évora, Portugal). Disponível em http://hdl.handle.net/10174/15920

Gorard, S. (Ed.). (2020). Getting Evidence into Education Evaluating the Routes to Policy and Practice. London: Routledge.

Gustafsson, J. E., Ehren, M. C. M., Conyngham, G., McNamara, G., Altrichter, H. e O'Hara, J. (2015). From inspection to quality: Ways in which school inspection influences change in schools. Studies in Educational Evaluation, 47, 47-57. doi: 10.1016/j.stueduc.2015.07.002

Hargreaves, A. e Fullan, M. (2012). Professional capital: Transforming teaching in every school. New York: Teachers College Press.

Hult, A. e Edström, C. (2016). Teacher ambivalence towards school evaluation: promoting and ruining teacher professionalism. Education Inquiry, ス3), 305325. doi: 10.3402/edui.v7.30200

Imants, J. e Van der Wal, M. M. (2019). A model of teacher agency in professional development and school reform. Journal of Curriculum Studies, 52(1), 1-14. doi: 10.1080/00220272.2019.1604809 
Inspeção Geral da Educação e Ciência. (2008). Avaliação Externa das Escolas Ciclos de Avaliação. Disponível em https://bit.ly/3dp3iXf

Inspeção Geral da Educação e Ciência. (2019). Terceiro Ciclo da Avaliação Externa das Escolas - Metodologia. Disponível em https://bit.ly/37T7YUI

Jones, K. L., Tymms, P., Kemethofer, D., O’Hara, J., McNamara, G., Huber, S., Myrberg, E., Skedsmo, G. e Greger, D. (2017). The unintended consequences of inspection: the prevalence of inspection side-effects in Austria, the Czech Republic, England, Ireland, the Netherlands, Sweden, and Switzerland. Oxford Review of Education, 43, 1-18. doi: 10.1080/03054985.2017.1352499

Kemethofer, D., Gustafsson, J. e Altrichter, H. (2017). Comparing effects of school inspections in Sweden and Austria. Education Assessment, Evaluation and Accountability, 29(4), 319-337. doi: 10.1007/s11092-017-9265-1

Leijen, Ä., Pedaste, M. e Lepp, L. (2019). Teacher agency following the ecological model: how it is achieved and how it could be strengthened by different types of reflection. British Journal of Educational Studies, 1(16). doi: 10.1080/00071005.2019.1672855

Merton, R. K. (1973). The normative structure of science. Em N.W. Storer (Ed.), The sociology of science: Theoretical and empirical investigations (pp. 267-278). Chicago: University of Chicago Press.

Pacheco, J. A., Morgado J. C. e Sousa, J. (Eds.). (2020). Avaliação institucional: perspetivas teórico-conceptuais. Porto: Porto Editora.

Morris, M. (2003). Ethical Considerations in Evaluation. Em T. Kellaghan \& D. L. Stufflebeam (Eds.), International Handbook of Educational Evaluation (pp. 303327). Dordrecht: Kluwer Academic Publishers.

Oliveira, D. (2017). Qualidade da educação em Portugal: o papel da avaliação externa de escolas. (Tese de Doutoramento, Universidade de Aveiro). Aveiro: Universidade de Aveiro.

Pacheco, J. A. (2016). Resultados globais do Projeto. In C. Barreira, M. Bidarra, \& M. Vaz-Rebelo (Org.), Estudos sobre Avaliação Externa de Escolas (pp. 262-271). Porto: Porto Editora. 
Pacheco, J. A. (Org.). (2014). Avaliação Externa de Escolas: Quadro Teórico/Conceptual. Porto: Porto Editora.

Pascual, J. (2019). Innovación Educativa: Un proceso construido sobre relaciones de poder. Revista Educación, Política y Sociedad, 4(2), 9-30.

Penninckx, M. (2017). Effects and side effects of school inspections: a general framework. Studies in Educational Evaluation, 52, 1-11. doi: 10.1016/j.stueduc.2016.06.006

Penninckx, M. e Vanhoof, J. (2015). Insights gained by schools and emotional consequences of school inspections. School Leadership and Management, 35(5), 477-501. doi: 10.1080/13632434.2015.1107036

Priestley, M., Biesta, G. J. J. e Robinson, S. (2015). Teacher Agency: An Ecological Approach. London: Bloomsbury Academic.

Quintelier, A., Vanhoof, J. e De Maeyer, S. (2018). Understanding the influence of teachers' cognitive and effective responses upon school inspection feedback acceptance. Education Assessment, Evaluation and Accountability, 30(4), 399431. doi: 10.1007/s11092-018-9286-4

Russell, B. (2009). Bertrand Russell Autobiography. New York: Routledge.

Sampaio, M. (2018). Avaliação externa de escolas e programa TEIP: que lugar(es) para a justiça social? (Tese de Doutoramento, Universidade do Porto). Disponível em https://bit.ly/2Nki4nA

Shah, H. (2020). Global problems need social science. Nature, 57716), 295. doi: $10.1038 / \mathrm{d} 41586-020-00064-\mathrm{x}$

Silvestre, M. J. (2013). Avaliação das escolas. Avaliação nas escolas. (Tese de Doutoramento, Universidade de Évora, Portugal). Disponível em https://bit.ly/2Yp0rt7

Sousa, J. (2019). Conceptualizar o currículo da educação pré-escolar no contexto da Avaliação Externa das Escolas em Portugal (Tese de Doutoramento, Universidade do Minho). Braga: Universidade do Minho. Disponível em https://bit.ly/31fKBDb 
Stake, R. (2006). Evaluación comprensiva y evaluación basada en estándares. Barcelona: Graó.

Stufflebeam, D. L. (2003). Professional Standards and Principles for Evaluations. In T. Kellaghan \& D. L. Stufflebeam (Eds.), International Handbook of Educational Evaluation (pp. 279-302). Dordrecht: Kluwer Academic Publishers.

Vincent-Lancrin, S., Urgel, J., Kar, S. e Jacotin, G. (2019). Measuring Innovation in Education 2019: What Has Changed in the Classroom?. Paris: OECD Publishing. Vo, A. T. e Christie, C.A. (2018). Where impact measurement meets evaluation: tensions, challenges, and opportunities. American Journal of Evaluation, 39(2), 383-388. doi: $10.1177 / 1098214018778813$

Warren, A. N. e Ward, N. A. (2017). 'This is my new normal': teachers' accounts of evaluation policy at local school board meetings. Journal of Education Policy, 33(6), 840-860. https://doi.org/10.1080/02680939.2017.1390164

Worthen, B. R. e Sanders, J. R. (1987). Educational Evaluation. New York: Longman.

Recebido em: 4 de março de 2020

Aceito em: 5 de junho de 2020

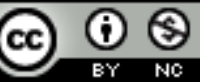

Revista Educación, Política y Sociedad (ISSN 2445-4109) está distribuida bajo una Licencia Creative Commons Atribución-NoComercial 4.0 Internacional 